\title{
Low-complexity multiuser MIMO downlink system based on a small-sized $\mathrm{CQI}$ quantizer
}

\author{
Jiho Song ${ }^{1}$, Jong-Ho Lee ${ }^{2}$, Seong-Cheol Kim ${ }^{1}$ and Younglok Kim ${ }^{3 *}$
}

\begin{abstract}
It is known that the conventional semi-orthogonal user selection based on a greedy algorithm cannot provide a globally optimal solution due to its semi-orthogonal property. To find a more optimal user set and prevent the waste of the feedback resource at the base station, we present a multiuser multiple-input multiple-output system using a random beamforming (RBF) scheme, in which one unitary matrix is used. To reduce feedback overhead for channel quality information (CQI), we propose an efficient CQI quantizer based on a closed-form expression of expected SINR for selected users. Numerical results show that the RBF with the proposed CQI quantizer provides better throughput than conventional systems under minor levels of feedback.
\end{abstract}

\section{Introduction}

The study of multiuser multiple-input multiple-output (MU-MIMO) has focused on broadcast downlink channels as a promising solution to support high data rates in wireless communications. It is known that the MUMIMO system can serve multiple users simultaneously with reliable communications and that it can provide higher data rates than the point-to-point MIMO system owing to multiuser diversity [1-3]. In particular, dirty paper coding (DPC) has been shown to achieve high data rates that are close to the capacity upper bound $[4,5]$. However, this technique is based mainly on impractical assumption such as perfect knowledge of the wireless channel at the transmitter. To send the channel state information (CSI) back to the transmitter perfectly, considerable wireless resources are required to assist the feedback link between the base station (BS) and the mobile station (MS). This adds a high level of complexity to the communication system, which is not feasible in practice.

Numerous studies have investigated and designed MU-MIMO systems that operate reliably under limited knowledge of the channel at the transmitter [6-9]. The semi-orthogonal user selection (SUS) algorithm in [6] shows a simple MU-MIMO system with zero-forcing beamforming (ZFBF) [10] and limited feedback [11,12]. Although this system achieves a sum-rate close to the

\footnotetext{
* Correspondence: ylkim@sogang.ac.kr

${ }^{3}$ Department of Electronic Engineering, Sogang University, Seoul, Korea Full list of author information is available at the end of the article
}

DPC in the regime of large number of users, the overall performance is restricted seriously by a quantization error due to the mismatch between the predefined code and the normalized channel. For this reason, antenna combining techniques have been developed that decrease this quantization error using multiple antennas at the MS $[7,8]$. However, the SUS algorithm based on the conventional greedy algorithm does not guarantee a globally optimized user set. Furthermore, in earlier research, quantizing the channel quality information (CQI) is not considered.

In this article, we consider a MU-MIMO downlink system with minor levels of feedback in which each user sends channel direction information (CDI) quantized by a $\log _{2} M$-sized codebook instead of by the large predefined CDI codebook used in SUS. Furthermore, to reduce the feedback overhead for CQI, we propose a small-sized CQI quantizer based on the closed-form expression of the CQI of selected users. It is shown that the proposed quantizer provides a point of reference for the quantizing boundaries of CQI feedback and reflects the sum-rate growth resulting from multiuser diversity with only 1 or 2 bits. The proposed CQI quantizer operates well with minor levels of feedback.

The remainder of this article is organized as follows. In Section 2, we introduce the system model and propose a low-complexity and small-sized feedback multiantenna downlink system which is based on the random beamforming (RBF) scheme in [13]. In Section 3, we present the user selection algorithm in the RBF scheme 
and we review the SUS algorithm and improve upon its weaknesses. In Section 4, the closed form expression for CQI is proposed when $N=M$ or $N \neq M$ respectively in order to set up the criteria of quantizing CQI. In Section 5, the numerical results are presented and Section 6 details our conclusions.

\section{System model and the proposed system}

We consider a single-cell MIMO downlink channel in which the BS has $M$ antennas and each of $K$ users has $N$ antennas located within the BS coverage area. The channel between the BS and the MS is assumed to be a homogeneous and Rayleigh flat fading channel that has circularly symmetric complex Gaussian entries with zero-mean and unit variance. In this system, we assume that the channel is frequency-dependent and the MS experiences slow fading. Therefore, the channel coherence time is sufficient for sending the channel feedback information within the signaling interval. In addition, we assume that the feedback information is reported through an error-free and nondelayed feedback channel.

The received signal for the $k$ th user is represented as

$$
\bar{y}_{k}=H_{k} W \bar{s}+\bar{n}_{k}, \quad k=1, \ldots, K
$$

where $H_{k}=\left[\bar{h}_{k, 1}^{T}, \bar{h}_{k, 2}^{T}, \ldots, \bar{h}_{k, N}^{T}\right]^{T} \in \mathbf{C}^{N \times M}$ is a channel matrix for each user and $\bar{h}_{k, n} \in \mathbf{C}^{1 \times M}$ is a channel gain vector with zero-mean and unit variance for the $n$th antenna of the $k$ th user. $W=\left[\bar{w}_{1}, \ldots, \bar{w}_{M}\right] \in \mathrm{C}^{M \times M}$ is a ZFBF matrix for the set of selected users $S, \bar{n}_{k} \in \mathrm{C}^{N \times 1}$ is an additive white Gaussian noise vector with the covariance of $I_{N}$, where $I_{N}$ denotes a $N \times N$ identity matrix. $\bar{s}=\left[s_{\pi(1)}, \ldots, s_{\pi(M)}\right]^{T}$ is the information symbol vector for the selected set of users $S=\{\pi(1), \ldots, \pi(M)\}$ and $\bar{x}=W \bar{s}=\sum_{i=1}^{M} \bar{w}_{i} s_{\pi(i)}$ is the transmit symbol vector that is constrained by an average constraint power, $E\left\{\|\bar{x}\|^{2}\right\}=P$. $\bar{y}_{k}$ is the received signal vector at user $k$.

\subsection{Proposed MU-MIMO system}

In this section, we present a low-complexity and smallsized feedback multiple-antenna downlink system. The proposed system is based on the RBF scheme in [13] using only one unitary matrix - identity matrix $I_{M}$. (This is identical to the per user unitary and rate control $\left(\mathrm{PU}^{2} \mathrm{RC}\right)$ scheme in [14] which uses only one pre-coding matrix $I_{M}$.) For this reason, it is not necessary for each user to send preferred matrix index (PMI) feedback to the BS. In the proposed system, each MS has multiple antennas and an antenna combiner such as the quantization-based combining (QBC) in [7] or the maximum expected SINR combiner (MESC) in [8] is used. The received signal $y_{k, a}^{\text {eff }}$ after post-coding with an antenna combiner $\tilde{\eta}_{k, a}^{H} \in \mathbf{C}^{1 \times N}$ is given by

$$
\begin{aligned}
\gamma_{k, a}^{\text {eff }} & =\tilde{\eta}_{k, a}^{H} \bar{y}_{k}=\tilde{\eta}_{k, a}^{H} H_{k} W \bar{s}+\tilde{\eta}_{k, a}^{H} \bar{n}_{k,} \quad(1 \leq a \leq M, \quad 1 \leq k \leq K) \\
& =\tilde{\eta}_{k, a}^{H} H_{k} \bar{w}_{k} s_{k}+\tilde{\eta}_{k, a}^{H} H_{k} \sum_{\substack{i \in S \\
i \neq k}} \bar{w}_{i} s_{i}+\tilde{\eta}_{k, a}^{H} \bar{n}_{k} .
\end{aligned}
$$

We assume that perfect channel information is available at each MS and that this channel information is fed back to the BS using a feedback link. After computing all M CQIs, the MS feeds back one maximum CQIs to the BS. In this work, CQIs are quantized by the proposed quantizer with 1 or 2 bits.

With the CQIs from $K$ users, the BS constructs the selected user set and sends the feed-forward signal through the forward channels. The feed-forward signal contains information about which users will be served and which codebook vector is allocated to each selected user. With the feed-forward signal, selected users are able to construct proper combining vectors. The proposed RBF system illustrated in Figure 1 is described as follows.

(1) Each user computes the direction of the effective channel for $\mathrm{QBC}$ in [7] using all code vectors $\bar{c}_{a}$ (ath row of the identity matrix $I_{M}, 1 \leq a \leq M$ ) and normalizes the effective channel.

$$
\begin{aligned}
& \bar{h}_{k, a}^{\text {eff }}=\bar{c}_{a} Q_{k}^{H} Q_{k}, \quad(1 \leq a \leq M, \quad 1 \leq k \leq K) \\
& \tilde{h}_{k, a}^{\text {eff }}=\frac{\bar{h}_{k, a}^{\text {eff }}}{\left\|\bar{h}_{k, a}^{\text {eff }}\right\|}
\end{aligned}
$$

where $Q_{k} \doteq\left[\bar{q}_{1}^{T}, \ldots, \bar{q}_{N}^{T}\right]^{T}$

$\bar{q}_{x} \in \mathrm{C}^{1 \times M}$ : orthonormal basis for span $\left(H_{k}\right)$

$\|\bar{x}\|=\|\bar{x}\|_{2}:=\sqrt{\bar{x} \bar{x}^{H}}:$ vector norm $(2-$ norm $)$

(2) The combining vectors for QBC and MESC in $[7,8]$ are computed and then normalized to unit vector.

$$
\begin{aligned}
& \left(\bar{\eta}_{k, a}^{H}\right)_{\mathrm{QBC}}=\tilde{h}_{k, a}^{\text {eff }}\left(H_{k}^{H}\right)\left(H_{k} H_{k}^{H}\right)^{-1}, \quad(1 \leq a \leq M, \quad 1 \leq k \leq K) \\
& \left(\bar{\eta}_{k, a}^{H}\right)_{\mathrm{MESC}}=\left[\left(I+B_{k}\right)^{-1} \sqrt{\rho} H_{k} \bar{c}_{a}^{T}\right]^{H}
\end{aligned}
$$

where $B_{k}=\rho\left[H_{k}\left(I-\bar{c}_{a}^{H} \bar{c}_{a}\right) H_{k}^{H}\right], \quad \rho=P / M$

$$
\tilde{\eta}_{k, a}^{H}=\frac{\bar{\eta}_{k, a}^{H}}{\left\|\bar{\eta}_{k, a}^{H}\right\|}
$$

(3) The expected SINR (CQI) in [6] is computed with every direction of the effective channel. The normalized effective channel of the $k$ th user with the ath effective channel $\tilde{h}_{k, a}^{\text {eff }}$ is given as follows:

$$
\mathrm{CQI}_{k, a} \doteq \gamma_{k, a}=E\left[\operatorname{SINR}_{k, a}\right]=\frac{\rho\left\|\tilde{\eta}_{k, a}^{H} H_{k}\right\|^{2} \cos ^{2} \theta_{k, a}}{1+\rho\left\|\tilde{\eta}_{k, a}^{H} H_{k}\right\|^{2} \sin ^{2} \theta_{k, a}} \text {. (6) }
$$




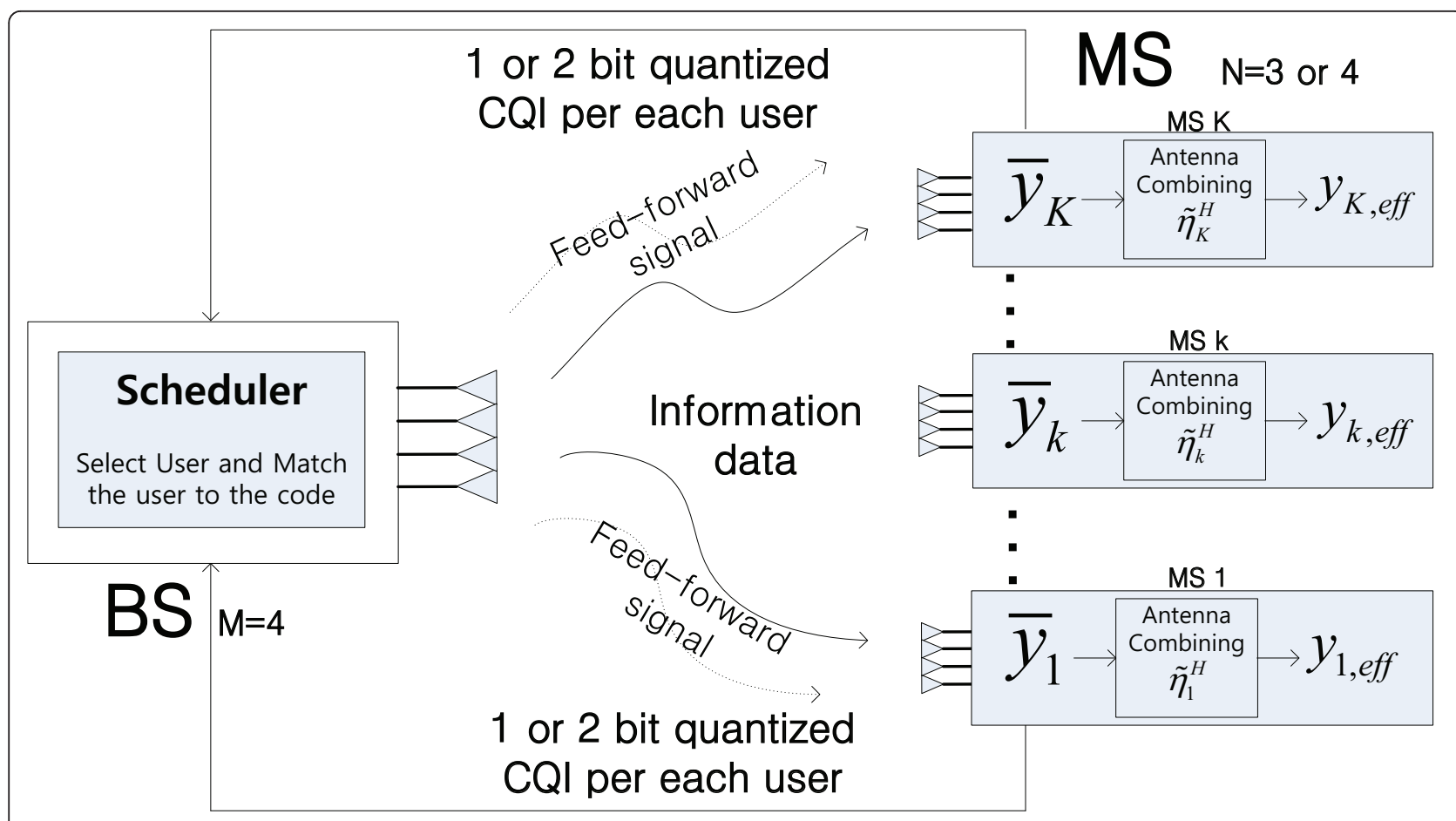

Figure 1 RBF MU-MIMO downlink system model.

where $\theta_{k, a}=\arccos \left(\left|\tilde{h}_{k, a}^{\text {eff }}-H_{a}^{H}\right|\right), \quad(1 \leq a \leq M, \quad 1 \leq k \leq K)$

$$
\bar{h}_{k, a}^{\text {eff }}=\tilde{\eta}_{k, a}^{H} H_{k}, \quad \tilde{h}_{k, a}^{\text {eff }}=\frac{\bar{h}_{k, a}^{\text {eff }}}{\left\|\bar{h}_{k, a}^{\text {eff }}\right\|}
$$

(4) Each user feeds back CDI and its related CQI to the BS according to the feedback scheme.

\section{User selection algorithm}

\subsection{User selection algorithm in RBF system}

In this section, we present the user selection algorithm with the CQI feedback matrix $F_{i} \in \mathbf{R}^{K \times M}(1 \leq i \leq M)$, which is made up of CQIs from each user. In the initial feedback matrix $F_{1}$, the $(k, a)$ th entry $C Q I_{k, a}$ represents the CQI feedback of the $k$ th user with the ath effective channel. The $C Q I_{k, a}$ that is used for user selection is described in (6).

(1) BS selects the first user $\pi(1)$ and the first effective channel code (1) simultaneously with the maximum entry from the entries of the initial feedback matrix $F_{1}$.

$$
\pi(1)=\arg \max _{1 \leq k \leq K} \mathrm{CQI}_{k, \sigma_{k},} \quad \operatorname{code}(1)=\bar{c}_{\sigma_{\pi(1)}}
$$

where

$\sigma_{k}=\arg \max _{1 \leq a \leq M} \mathrm{CQI}_{k, a} \quad$ for $1 \leq k \leq K, \quad \mathrm{CQI}_{k, a} \in F_{1}$
(2) The $(i+1)$ th feedback matrix $F_{i+1}$ is constructed by removing the entries of the $i$ th users $\pi(i)$ and the entries of the $i$ th effective channels code $(i)$ from the $i$ th feedback matrix. After doing this, the BS selects the $(i+1)$ th user and the effective channel with the maximum entry from the feedback matrix $F_{i+1}$ in (8). This user selection process is repeated until the BS constructs a selected set of users $S=\{\pi(1), \ldots, \pi(M)\}$ up to $M$.

$$
\text { let }\left(\mathrm{CQI}_{k, a} \in F_{i+1}\right)=0
$$

when $k=\pi(j)$ or $a=\sigma_{\pi(j)}, 1 \leq j \leq i$

$$
\pi(i+1)=\arg \max _{1 \leq k \leq K} \mathrm{CQI}_{k, \sigma_{k^{\prime}}} \quad \operatorname{code}(i+1)=\bar{c}_{\sigma_{\pi(i+1)}}
$$

where $\sigma_{k}=\arg \max _{1 \leq a \leq M} \operatorname{CQI}_{k, a}$ for $1 \leq k \leq K, \quad \mathrm{CQI}_{k, a} \in F_{i+1}$

\subsection{Modified SUS}

In this section, we review the SUS algorithm [6] and modify it to overcome its vulnerable aspects. In the SUS-based MU-MIMO system, the codebook design is based on the random vector quantization (RVQ) scheme in $[15,16]$. The predefined codebook, $C=\left\{\bar{c}_{1}, \ldots, \bar{c}_{2^{B} \mathrm{CDI}}\right\}$ of size $L=2^{B \mathrm{CD} \text {, }}$ is composed of $L$ isotropically distributed unit-norm codewords in $\mathrm{C}^{1 \times M}$, where $B_{\mathrm{CDI}}$ denotes the number of feedback bits for a single CDI. In the SUS algorithm, the BS tries to select users up to $M$ out of $K$ users. The BS selects the first user $\pi(1)=\arg \max _{k \in A_{1}} C Q Q I_{k, \sigma_{k}}$ which has the 
largest CQI out of the initial user set $A_{1}=\{1, \ldots, K\}$. The value of $\mathrm{CQI}_{k, \sigma_{k}}\left(\sigma_{k}=\arg \max _{1 \leq a \leq 2^{B} \mathrm{CDI}} \mathrm{CQI}_{k, a}\right.$ for $\left.\quad 1 \leq k \leq K\right)$ is described in (6) according to the antenna combiner. The BS constructs the user set,

$$
A_{i+1}=\left\{1 \leq k \leq K:\left|\hat{h}_{k} \hat{h}_{\pi\{j\}}^{H}\right| \leq \varepsilon, \quad 1 \leq j \leq i\right\}
$$

where $\hat{h}_{k}=\tilde{h}_{k, \sigma_{k}}^{\text {eff }}$ is a quantized effective channel vector of user $k$, and selects the $(i+1)$ th user $\pi(i+1)$ out of the user set $A_{i+1}$. In this formulation, the system design parameter $\varepsilon$, which determines the upper bound of the spatial correlation between quantized channels, is the critical parameter for the user selection. When the design parameter is set to a small value or when few users are located within the BS coverage area, user set $A_{i+1}$ can potentially be an empty set for some cases in which $i \leq M$, resulting no selection of the $(i+1)$ th user by the BS.

For this reason, we develop a modified SUS algorithm denoted as SUS-epsilon expansion (SUS-ee). In SUS-ee, the system increases the design parameter gradually until user set $A_{i+1}$ is not an empty set so as to guarantee the achievement of the multiplexing gain $M$.

With the modified user set denoted as,

$$
\begin{aligned}
& A_{i+1}^{e e}=\left\{1 \leq k \leq K:\left|\hat{h}_{k} \hat{h}_{\pi\{j\}}^{H}\right| \leq \varepsilon^{e e}, \quad 1 \leq j \leq i\right\} \\
& \pi(i+1)=\arg \max _{k \in A_{i+1}^{e e}} \mathrm{CQI}_{k, \sigma_{k}}
\end{aligned}
$$

the BS selects the next user $\pi(i+1)$. In this formulation, $\varepsilon^{e e}$ is an expanded design parameter. With the proposed algorithm, the BS can construct a selected set of users $S=\{\pi(1), \ldots, \pi(M)\}$ with cardinality up to $M$.

\section{Proposed CQI quantizer}

In the MU-MIMO downlink system, the CQI quantizer is also a critical factor determining the size of overall feedback. In this section, we derive the closed form expression of the CQI of selected users in order to quantize CQI with small bits. Then, we propose a CQI quantizer to better reflect the multiuser diversity. The proposed quantizer is derived for $\mathrm{QBC}$ because the distribution of the $\mathrm{CQI}$ resulting from $\mathrm{QBC}$ can be obtained analytically and is more amenable to analysis than MESC.

\section{1 $N=M$ : Closed form expression for CQI and the proposed quantizer}

\subsubsection{CQI quantizer under $Q B C$}

In the RBF system, identity matrix $I_{M}$ is considered as a codebook of $\log _{2} M$ bit size. When $N=M$, the combining vector is given in the shape of the row vector of the pseudo inverse channel matrix.

$$
\begin{gathered}
\bar{\eta}_{k, a}^{H}=\tilde{h}_{k, a}^{\text {eff }}\left(H_{k}^{H}\right)\left(H_{k} H_{k}^{H}\right)^{-1}=\tilde{h}_{k, a}^{\text {eff }}\left[\begin{array}{llll}
h_{11}^{i} & h_{12}^{i} & h_{13}^{i} & h_{14}^{i} \\
h_{21}^{i} & h_{22}^{i} & h_{23}^{i} & h_{24}^{i} \\
h_{31}^{i} & h_{32}^{i} & h_{33}^{i} & h_{34}^{i} \\
h_{41}^{i} & h_{42}^{i} & h_{43}^{i} & h_{44}^{i}
\end{array}\right]_{k} \\
=\text { ath row of }\left(H_{k}^{H}\right)\left(H_{k} H_{k}^{H}\right)^{-1} .
\end{gathered}
$$

With the combining vector, the CQI can be represented as the product of an equally allocated power $\rho$ and a norm of effective channel $\left\|\bar{h}_{k, a}^{\text {eff }}\right\| \|^{2}$ since there is no CDI quantization error when $N=M$. The CQI feedback of the $k$ th user with the ath effective channel is described as given by

$$
\begin{aligned}
\mathrm{CQI}_{k, a}= & \rho\left\|\tilde{\eta}_{k, a}^{H} H_{k}\right\|^{2}=\rho\left\|\bar{h}_{k, a}^{\mathrm{eff}}\right\|^{2} \\
& =\rho \mid \frac{\bar{\eta}_{k, a}^{H}}{\left\|\bar{\eta}_{k, a}^{H}\right\|} \times a \text { th column of }\left.H_{k}\right|^{2} \\
= & \frac{\rho}{\left\|\bar{\eta}_{k, a}^{H}\right\|^{2}}=\frac{\rho}{\sum_{l=1}^{M}\left|h_{a, l}^{i}\right|^{2}} \\
& =\frac{\rho}{\sum_{l=1}^{M}\left\{\left(\Re\left[h_{a, l}^{i}\right]\right)^{2}+\left(\mathfrak{F}\left[h_{a, l}^{i}\right]\right)^{2}\right\}} .
\end{aligned}
$$

As shown in (14), the CQI is related to the distribution of entries of the inverse channel matrix. According to $[7,17],\left\|\bar{h}_{k, a}^{\text {eff }}\right\|^{2}$ follows Chi-square distribution with variance $\sigma^{2}\left(\left\|\bar{h}_{k, a}^{\text {eff }}\right\|^{2} \sim \chi_{2(M-N+1)}^{2}\right)$ and the cdf is described as

$$
F_{X}(x)=1-e^{-\frac{x}{2 \sigma^{2}}}, \quad x \geq 0
$$

where $\sigma^{2}=\sigma_{q b c}^{2}=0.5$

By substituting $\frac{x}{2 \sigma^{2}}$ with $y, X$ and $Y$ follow the relation $X=2 \sigma^{2} Y$. Then, the distribution of $Y$ follows the type (iii) distribution in [[18], Theorem 4].

$$
F_{Y}(\gamma)=1-e^{-\gamma}, \quad y \geq 0
$$

In that case, the approximated $y$ can be obtained through the study of extreme value theory from order statistics. According to $[18,19]$, the distribution of $Y$ satisfies following inequality

$$
\operatorname{Pr}\left(\left|Y_{a: Q_{a}}-b_{Q_{a}}\right| \leq \log \log \sqrt{Q_{a}}\right) \geq 1-O\left(\frac{1}{\log Q_{a}}\right)(17)
$$

where $a_{Q_{a}}=1, b_{Q_{a}}=\log Q_{a}$ and $Q_{a}$ is the number of antennas in the ath user selection process.

When $Q_{a}$ is large enough, $y$ satisfies the following approximated formulation,

$$
y_{a: Q_{a}} \cong \log Q_{a}+O\left(\log \log Q_{a}\right)
$$




$$
\begin{aligned}
& x_{a: Q_{a}} \cong 2 \sigma^{2}\left(\log Q_{a}\right) \\
& \mathrm{CQI}_{a: Q_{a}} \doteq \gamma_{a: Q_{a}} \cong 2 \sigma^{2} \rho\left(\log Q_{a}\right) .
\end{aligned}
$$

$$
\text { where } \sigma^{2}=\sigma_{q b c}^{2}=0.5
$$

where $\gamma_{a: Q_{a}}$ in (20) is the approximated value of the CQI when $N=M$ and $Q_{a}$ is the number of antennas in the $a$ th user selection process. $Q_{a}$ used under RBF and SUS-ee system will be presented in the Section 4.3.

\subsubsection{CQI quantizer under MESC}

While the distribution of the $\left\|\bar{h}_{k, a}^{\text {eff }}\right\| \|^{2}$ under QBC can be obtained analytically, it is hard to analyze the distribution of the $\left\|\bar{h}_{k, a}^{\text {eff }}\right\|^{2}$ under MESC. For this reason, we describe the distribution of the $\left\|\bar{h}_{k, a}^{\text {eff }}\right\| \|^{2}$ under MESC using numerical results. According to the numerical results of Monte-Carlo simulation, we assume that $\left\|\bar{h}_{k, a}^{\text {eff }}\right\|^{2}$ has a Chi-square distribution with variance $\sigma^{2}$ defined by

$$
\sigma^{2}=\sigma_{\text {mesc }}^{2}= \begin{cases}0.7, & \rho \leq 1(\mathrm{~dB}) \\ 0.7 \rho^{-0.1}, & 1<\rho \leq 28(\mathrm{~dB}) \\ 0.5, & \rho>28(\mathrm{~dB})\end{cases}
$$

\section{$4.21<N<M$ : Closed form expression for CQI and the proposed quantizer}

In this section, we develop the closed form expression of the CQI of selected users when $N \neq M$. In the case of $N$ $\neq M$, removing the quantization error between the codeword and the effective channel completely is not possible. To develop the closed form expression of the CQI of selected users, we need to derive the cdf of the CQI. For this reason, we must know the distribution of both the norm of the effective channel $\left\|\bar{h}_{k, a}^{\text {eff }}\right\|^{2}$ and the quantization error term $\sin ^{2} \theta_{k, a}$ As explained in Section 4.1, the norm of the effective channel $\left\|\bar{h}_{k, a}^{\text {eff }}\right\|^{2}$ has a Chisquare distribution $\left(\left\|\bar{h}_{k, a}^{\text {eff }}\right\|^{2} \sim \chi_{2(M-N+1)}^{2}\right)$. In addition, according to [7], quantization error $\sin ^{2} \theta_{k, a}$ follows the approximated formulation as given by

$$
F_{\sin ^{2} \theta_{k, a}}(x) \cong \begin{cases}\left(\begin{array}{l}
M-1 \\
N-1
\end{array}\right) x^{M-N}, & (0 \leq x \leq \delta) \\
1, & (x>\delta)\end{cases}
$$

where

$$
\delta= \begin{cases}\frac{1}{\left(\begin{array}{c}
M-1 \\
N-1
\end{array}\right)}, & (N=M-1) \\
\frac{1}{\sqrt{(M-1} \begin{array}{c}
M-1 \\
N-1
\end{array}},(N=M-2)\end{cases}
$$

With the distribution of $\left\|\bar{h}_{k, a}^{\text {eff }}\right\|^{2}$ and $\sin ^{2} \theta_{k, a}$, we derive the cdf of CQI in the same way as in [[6], Section 5: $N=1$ ]. At first, we derive the distribution of the interference term in Lemma 1 and it is proved in Appendix 1.

Lemma 1: (Interference term)

$$
\left\|\bar{h}_{k, a}^{\text {eff }}\right\|^{2} \sin ^{2} \theta_{k, a} \sim \operatorname{Gamma}\left(M-N, 2 \sigma^{2} \delta\right) \sim 2 \sigma^{2} \delta Y=I
$$

where

$$
\begin{aligned}
& Y \sim \operatorname{Gamma}(M-N, 1) \\
& \sigma^{2}: \text { Variance of }\left\|\bar{h}_{k, a}^{\text {eff }}\right\|^{2} \quad\left(\sigma_{q b c}^{2} \text { in }(15) \text { and } \sigma_{\text {mesc }}^{2} \text { in }(21)\right)
\end{aligned}
$$

Proof: Appendix 1

As can be seen in Appendix 1, the interference term has a Gamma distribution, $\operatorname{Gamma}\left(M-N, 2 \sigma^{2} \delta\right)$.

Lemma 2: (Information signal term)

$$
\left\|\bar{h}_{k, a}^{\text {eff }}\right\|^{2} \cos ^{2} \theta_{k, a} \sim t(X+(1-\delta) Y)=S
$$

where

$X \sim \operatorname{Gamma}(1,1), Y \sim \operatorname{Gamma}(M-N, 1)$

$t=2 \sigma^{2}$

Proof: Appendix 2

In Appendix 2, to derive the distribution of $\left\|\bar{h}_{k, a}^{\text {eff }}\right\|^{2} \cos ^{2} \theta_{k, a}$, we verify that the joint distribution of $\left\|\bar{h}_{k, a}^{\text {eff }}\right\|^{2} \cos ^{2} \theta_{k, a}$ and $\left.\left\|\bar{h}_{k, a}^{\text {eff }}\right\|\right|^{2} \sin ^{2} \theta_{k, a}$ is comparable with the joint distribution of $I$ and $S$. Therefore, the information signal term can be described as the sum of the two Gamma variables $X$ and $Y$. Furthermore, it is shown that the distribution of $\gamma_{k, a}=\frac{\rho\left\|\bar{h}_{k, a}^{\text {eff }}\right\|^{2} \cos ^{2} \theta_{k, a}}{1+\rho|| \bar{h}_{k, a}^{\text {eff }} \|^{2} \sin ^{2} \theta_{k, a}}$ is equal to the distribution of $\gamma=\frac{\rho S}{1+\rho I^{1}}$.

Lemma 3: (CQI: Expected SINR)

Define

$$
\gamma=\frac{\rho S}{1+\rho I}=\frac{\rho t(X+(1-\delta) Y)}{1+\rho \delta t Y}
$$

then

$$
F_{\gamma}(x)=1-\frac{\left(\begin{array}{c}
M-1 \\
N-1
\end{array}\right) e^{-\frac{x}{2 \sigma^{2} \rho}}}{(x+1)^{M-N}}
$$

Proof: Appendix 3

Since it is proved that the distribution of $\gamma_{k, a}$ is equal to the distribution of $\gamma$, in Lemma 2, the cdf of $\gamma_{k, a}$ can be derived using the distribution of $\gamma$. In Lemma 3, we define $\gamma$ with two independent Gamma variables $X$ and $Y$. For this reason, the cdf of $\gamma$ can be derived using $X$ and $Y$.

Theorem 1: (Largest order statistic among CQIs for $Q_{a}$ candidates: using extreme value theory) 
For large $Q_{a}$

$$
\begin{aligned}
\mathrm{CQI}_{a: Q_{a}} \doteq \gamma_{a: Q_{a}} \cong & 2 \sigma^{2} \rho\left[\log \left[\frac{Q_{a}}{\left(2 \sigma^{2} \delta \rho\right)^{M-N}}\right]-\right. \\
& \left.(M-N) \log \left[\log \left[\frac{Q_{a}}{\left(2 \sigma^{2} \delta \rho\right)^{M-N}}\right]+\frac{1}{2 \sigma^{2} \rho}\right]\right]
\end{aligned}
$$

where $Q_{a}$ : The number of antennas in the $a$ th user selection process

Proof: Appendix 4

In Theorem 1, $\gamma_{a: Q_{a}}$ is the approximated value of the CQI when $1<N<M$. Since the cdf in Lemma 3 can be changed to follow the type (iii) distribution in [[18], Theorem 4], the closed form expression of $\mathrm{CQI}_{k, a}$ can be analyzed using the studies of extreme value theory when $N \neq M . Q_{a}$ used under the RBF and SUS-ee system will be presented in the next section.

\subsection{The number of antennas in the ath user selection process}

In this section, the number of user candidates in each user selection process are described. At first, $Q_{a}$ used in RBF is shown as

$$
Q_{a}=\left(Q_{a}\right)_{\mathrm{RBF}} \doteq(K-a+1)(M-a+1) \quad 1 \leq a \leq M .
$$

In contrast to the RBF, the number of user candidates used in the user selection stage under the SUS-ee algorithm is described as follows:

$$
\begin{array}{r}
Q_{a}=\left(Q_{a}\right)_{\text {SUS-ee }} \doteq\left[K-(a-1) \max \left(1, K / 2^{B_{C D I}}\right)\right] \alpha_{a} \\
{\left[2^{B_{C D I}}-(a-1)\right], \quad 1 \leq a \leq M}
\end{array}
$$

where $\alpha_{a}=\left\{\begin{array}{cc}1, & a=1 \\ I_{\mathcal{E}^{2}}(a-1, M-a+1), & a>1\end{array}\right.$.

Here, $I_{z}(x, y)$ is the regularized incomplete beta function which determines the size of the user pool, which varies according to the user selection order [10]. The constant $\alpha_{a}$ represents the probability that channel vectors of the user pool are in the set of vectors that are semi-orthogonal (referred to as $\varepsilon$-orthogonal in [6]) to all of the CDIs of the formerly selected users. As explained in the Section 3.2, the design parameter $\varepsilon$ is expanded in the modified SUS-ee algorithm and is assumed to be $\varepsilon^{e e}=\varepsilon+0.05$ in the fourth user selection stage according to the numerical results.

\subsection{CQI quantization boundary}

With the closed form expression of CQI, the quantization boundary of the CQI feedback is determined. In this work, we use 1 or 2 bit size CQI ( 2 or 4 level) quantizers. In the case of RBF based system, the CQI quantization boundaries are represented in Table 1. The CQI quantization boundaries in SUS-ee based system are represented in Table 2.

\subsection{Complexity analysis}

In this section, the complexity of the proposed RBF system is compared to that of a SUS-ee-based system. The complexity comparison is described in Table 3.

The RBF system is operated under low computational complexity at the BS stage because there is no need for vector computation in the user selection procedure and pre-coding operation at the beamformer, unlike in SUSee. In SUS-ee, BS has to let the selected users know their effective channel out of $2^{B_{\mathrm{CDI}}}$ effective channels, whereas the BS selects the feed-forward information for each selected user out of only $M$ effective channels in RBF. Furthermore, at the MS stage, each user has to compute only $\mathrm{M}$ CQIs in $\mathrm{RBF}$, whereas $2^{B_{\mathrm{CDI}}} \mathrm{CQIs}$ should be computed in SUS-ee. By decreasing the computational complexity at the BS, selecting users and allocating the desired information to each antenna can be performed more reliably within the signaling interval.

\section{Numerical results}

The numerical performances of the proposed system are discussed. We compared the numerical results of RBF to the results of three different MU-MIMO downlink systems (SUS-ee with antenna selection (AS) [6,7], QBC [7] and MESC [8]). The total size of the feedback used by each user is given in Table 4 .

\begin{tabular}{|c|c|c|}
\hline & 1-bit quantizer $(N=M)$ & 1-bit quantizer $(N=M-1)$ \\
\hline Level 1 & $0<x<0.85^{2} \gamma_{4: Q_{4}}$ & $0<x<0.85^{2} \gamma_{\kappa}: Q_{\kappa}(\mathrm{QBC}: \kappa=2, \mathrm{MESC}: \kappa=3)$ \\
\hline \multirow[t]{2}{*}{ Level 2} & $0.85^{2} \gamma_{4: Q_{4}} \leq x<\infty$ & $0.85^{2} \gamma_{\kappa}: Q_{\kappa} \leq x<\infty(\mathrm{QBC}: \kappa=2, \mathrm{MESC}: \kappa=3)$ \\
\hline & 2-bit quantizer $(N=M)$ & 2-bit quantizer $(N=M-1)$ \\
\hline Level 1 & $0<x<0.8^{2} \gamma_{4: Q_{4}}$ & $0<x<0.8^{2} \gamma_{3: Q_{3}}$ \\
\hline Level 2 & $0.8^{2} \gamma_{4: Q_{4}} \leq x<0.85^{2} \gamma_{3: Q_{3}}$ & $0.8^{2} \gamma_{3: Q_{3}} \leq x<0.9^{2} \gamma_{2: Q_{2}}$ \\
\hline Level 3 & $0.85^{2} \gamma_{3: Q_{3}} \leq x<0.9^{2} \gamma_{1: Q_{1}}$ & $0.9^{2} \gamma_{2: Q_{2}} \leq x<\gamma_{1: Q_{1}}$ \\
\hline Level 4 & $0.9^{2} \gamma_{1: Q_{1}} \leq x<\infty$ & $\gamma_{1: Q_{1}} \leq x<\infty$ \\
\hline Expected & $\gamma_{a: Q_{a}}=2 \sigma^{2} \rho \log Q_{a}$ & $\gamma_{a: Q_{a}}=2 \sigma^{2} \rho\left[\log \left(\frac{\left(\begin{array}{c}3 \\
2\end{array}\right) Q_{a}}{2 \sigma^{2} \rho}\right)-\log \left[\log \left(\frac{\left(\begin{array}{c}3 \\
2\end{array}\right) Q_{a}}{2 \sigma^{2} \rho}\right)+\frac{1}{2 \sigma^{2} \rho}\right]\right.$ \\
\hline
\end{tabular}

First, Figure 2 compares the results between the SUS and the SUS-ee algorithm under QBC when the system design parameter $\varepsilon$ is 0.3 . As shown in Figure 2, by

Table 1 The proposed CQI quantizer (RBF) 
Table 2 The proposed CQI quantizer (SUS-ee)

\begin{tabular}{lll}
\hline & 2-bit quantizer $(\boldsymbol{N}=\boldsymbol{M})$ & 2-bit quantizer $(\boldsymbol{N}=\boldsymbol{M}$ - 1) \\
\hline Level 1 & $0<x<0.7^{2} \gamma_{3: Q_{3}}$ & $0<x<0.9^{2} \gamma_{3: Q_{3}}$ \\
Level 2 & $0.7^{2} \gamma_{3: Q_{3}} \leq x<0.8^{2} \gamma_{2: Q_{2}}$ & $0.9^{2} \gamma_{3: Q_{3}} \leq x<0.95^{2} \gamma_{2: Q_{2}}$ \\
Level 3 & $0.8^{2} \gamma_{2: Q_{2}} \leq x<0.85^{2} \gamma_{1: Q_{1}}$ & $0.95^{2} \gamma_{2: Q_{2}} \leq x<\gamma_{1: Q_{1}}$ \\
Level 4 & $0.85^{2} \gamma_{1: Q_{1}} \leq x<\infty$ & $\gamma_{1: Q_{1}} \leq x<\infty$ \\
Expected & $\gamma_{a: Q_{a}}=2 \sigma^{2} \rho \log Q_{a}$ & $\gamma_{a: Q_{a}}=2 \sigma^{2} \rho\left[\log \left(\frac{\left(\begin{array}{l}3 \\
2\end{array}\right) Q_{a}}{2 \sigma^{2} \rho}\right)-\log \left[\log \left(\frac{\left(\begin{array}{l}3 \\
2\end{array}\right) Q_{a}}{2 \sigma^{2} \rho}\right)+\frac{1}{2 \sigma^{2} \rho}\right]\right]$ \\
CQl & & \\
\hline
\end{tabular}

adaptively increasing $\varepsilon$ in the SUS-ee algorithm, M users are serviced simultaneously and the sum-rate is increased by about $40 \%$ when $B_{\mathrm{CDI}}=8, K=30$ and $P=15 \mathrm{~dB}$.

Figures 3 and 4 plot the performance of the proposed CQI quantizer. The CQI quantizer shows better performance than the Lloyd-Max quantizer [20,21] as the number of user increases. Both the proposed quantizer for RBF and the SUS-ee algorithm can quantize CQI effectively and minimize performance degradation with both 1 and 2 bit CQI feedback. This is attributable to the fact that the proposed CQI quantizers is a function of the number of users and the distribution of the CQI, whereas the conventional quantizer is a function of only the distribution of the CQI. The proposed quantizer for RBF shows better performance than that for SUS-ee because the exact number of user candidates for SUS-ee cannot be determined.

In Figure 5, the sum-rate results from the numerical simulation and from formulation with a closed form for QBC or MESC are compared. With the closed form expression for CQI in Section 4, the sum-rate formulation can be represented as follows:

$$
R=\sum_{a=1}^{M} \log _{2}\left(1+\gamma_{a: Q_{a}}\right)
$$

where $\gamma_{a} Q_{a}$ is the expected SINR in (20) and (41, Appendix 4), for $N=M$ and $N=M-1$ case. As shown in (25), $R$ is the sum-rate which grows like $M \log _{2} \log Q$ due to multiplexing and multiuser diversity gains.
According to the assumption of a large user regime in the formulation with a closed form, when the number of users in the system is not large enough, a substantial difference between the numerical results and the expectation based on the closed form can be seen. However, as $K$ increases, the difference decreases to verify the accuracy of the formulation with a closed form.

In Figure 6, RBF shows better performance than SUSee-based systems under minor feedback conditions when $N=M$ or $N=M-1$. In these numerical simulations, with the QBC or MESC technique, SUS-ee system uses a 5-bit size codebook and with the AS technique, it uses a 6- and 8-bit size codebook. Although systems based on the SUS-ee have $2^{3}$ times more effective channel vectors for CQI than RBF, the user pool employed in the SUS-ee algorithm is determined entirely by the formerly selected users. If the previously selected users are not semi-orthogonal to the rest of the users, the number of user candidates in the next user selection stage will be highly restricted. Furthermore, if the effective channel vectors of the remaining users in the user selection stage are equal to the effective channel vectors of the previously selected users, these users will not have the opportunity to be serviced because each user feeds back only one CQI. Regardless of the fact that each user can fully remove the interference when $N=M$, the semi-orthogonality between the effective channel of users is a still critical issue of the system. By increasing the system design parameter $\varepsilon$, the effective channel gains for a set of selected users will be increased due to the multiuser diversity. However, the loss resulting from the normalization

Table 3 Complexity comparison between the RBF and the SUS-ee

\begin{tabular}{|c|c|c|}
\hline & RBF & SUS-ee \\
\hline \multicolumn{3}{|l|}{ BS } \\
\hline Pre-coding & N/A & $\mathrm{M} \times \mathrm{M}$ matrix inversion \\
\hline User selection & $\begin{array}{l}\text { Simple magnitude comparison between } \\
\text { quantized CQls from } K \text { users }\end{array}$ & $\begin{array}{l}\text { Vector computations are needed between the previously selected users and the } \\
\text { rest of the users until constructing users up to } M\end{array}$ \\
\hline $\begin{array}{l}\text { Feed-forward } \\
\text { information }\end{array}$ & $\begin{array}{l}\text { One desired effective channel out of } M= \\
4 \text { effective channels }\end{array}$ & One desired effective channel out of $2^{B_{\mathrm{CDI}}}$ effective channels \\
\hline \multicolumn{3}{|l|}{ MS } \\
\hline $\begin{array}{l}\text { Finding } \\
\text { feedback } \\
\text { information }\end{array}$ & $\begin{array}{l}\text { Compute } M=4 \text { combining vectors and } \\
\text { CQls }\end{array}$ & Compute $2^{B_{\mathrm{CDI}} \text { combining vectors and CQIs }}$ \\
\hline
\end{tabular}


Table 4 The size of feedback used in each MU-MIMO system

\begin{tabular}{lll}
\hline System & Feedback organization & $\begin{array}{l}\text { Total } \\
\text { feedback }\end{array}$ \\
\hline RBF w. QBC or MESC & CDI: 2 bits, CQI: 1 or 2 bits & 3 or 4 bits \\
SUS-ee w. QBC or & CDI: 5,6,7 or 8 bits, CQI: 2 & $7,8,9$ or 10 bits \\
MESC & bits & \\
\hline
\end{tabular}

process in ZFBF matrix $W$ (Moore-Penrose pseudoinverse matrix of set of selected users $S$ in [6]) also grows. For these reasons, SUS-ee does not guarantee that a globally optimized user set solution will be found. In RBF, the selected user set approaches a globally optimized solution because the effective channel vectors are completely orthogonal to each other. Additionally, RBF can guarantee the construction of a user set composed of up to $M$ users, even in a small user regime.

Figures 7 and 8 display the sum-rate vs. $K$ curves with power constraint $P$ as 10 or $20 \mathrm{~dB}$. In the figures, the RBF system is operated under 3 or 4 bit feedback conditions, whereas the SUS-ee system is operated under 9 or 10 bit feedback conditions in Figure 7 and under 7 or 8 bit feedback conditions in Figure 8, respectively. Despite the fact that the numerical results of the RBF performance are about $2.5 \mathrm{bps} / \mathrm{Hz}$ below that of SUS-ee with perfect CSIT in Figure 7, they still show better performance than SUS-ee-based systems, especially with a small number of users. For the best-case example, the sum-rate results of RBF are $4.5 \mathrm{bps} / \mathrm{Hz}$ higher than those of two different MU-MIMO systems when $K=8$ and $P=20 \mathrm{~dB}$ employing 4 bit feedback overall. As shown in Figures 7 and 8, while the size of all feedback for RBF with MESC (2 bit CQI) is 6 and 4 bits smaller than that of SUS-ee with MESC, respectively, the proposed system shows better throughput performance. With RBF (1 bit CQI), the system can achieve a reduction in the feedback overhead of up to 7 bits out of total 10 bits when $P=10 \mathrm{~dB}$ in Figure 7 . When $N$ is equal or similar to $M(N=4$ or 3$)$, the negative effect of a small candidate pool of effective channels

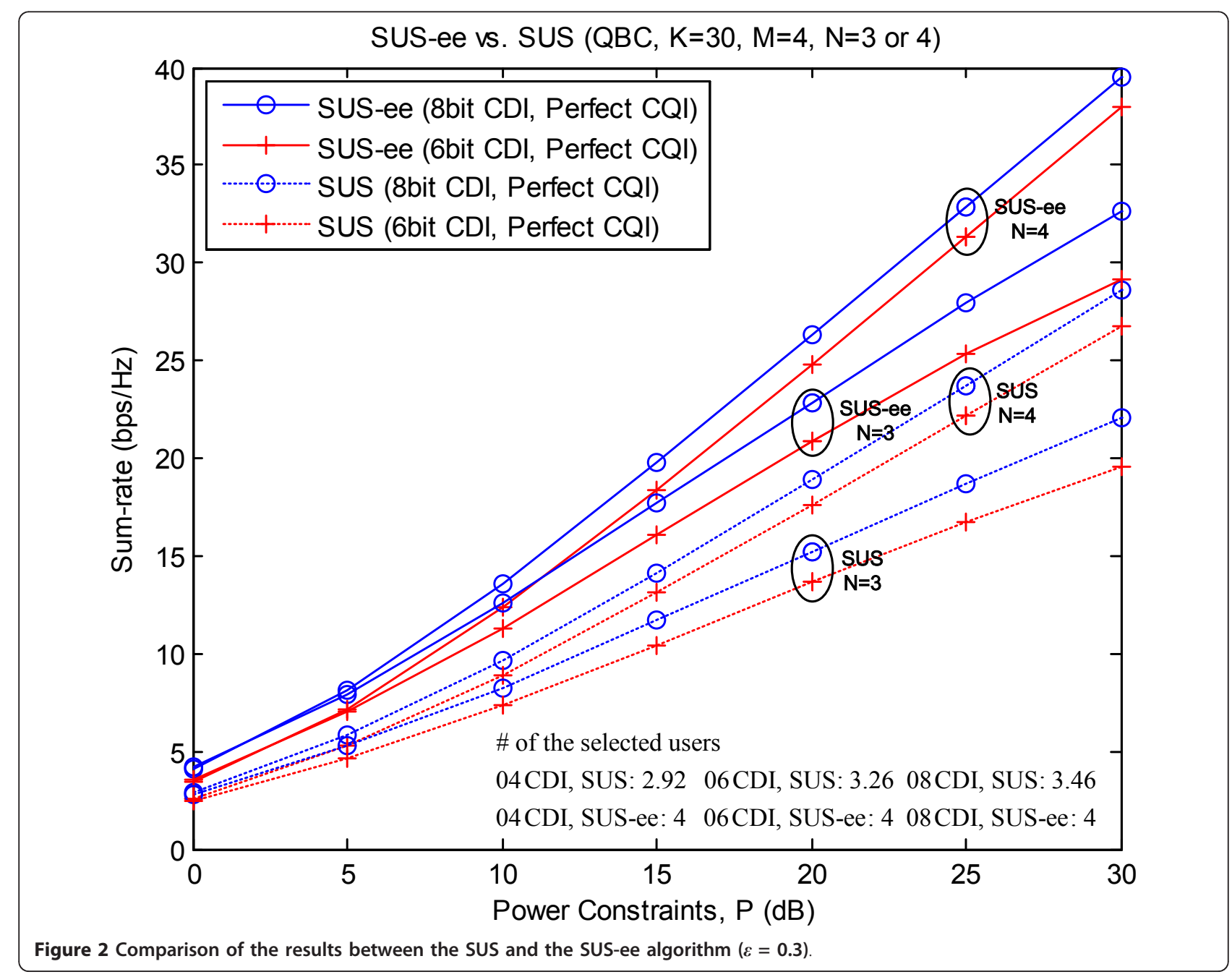




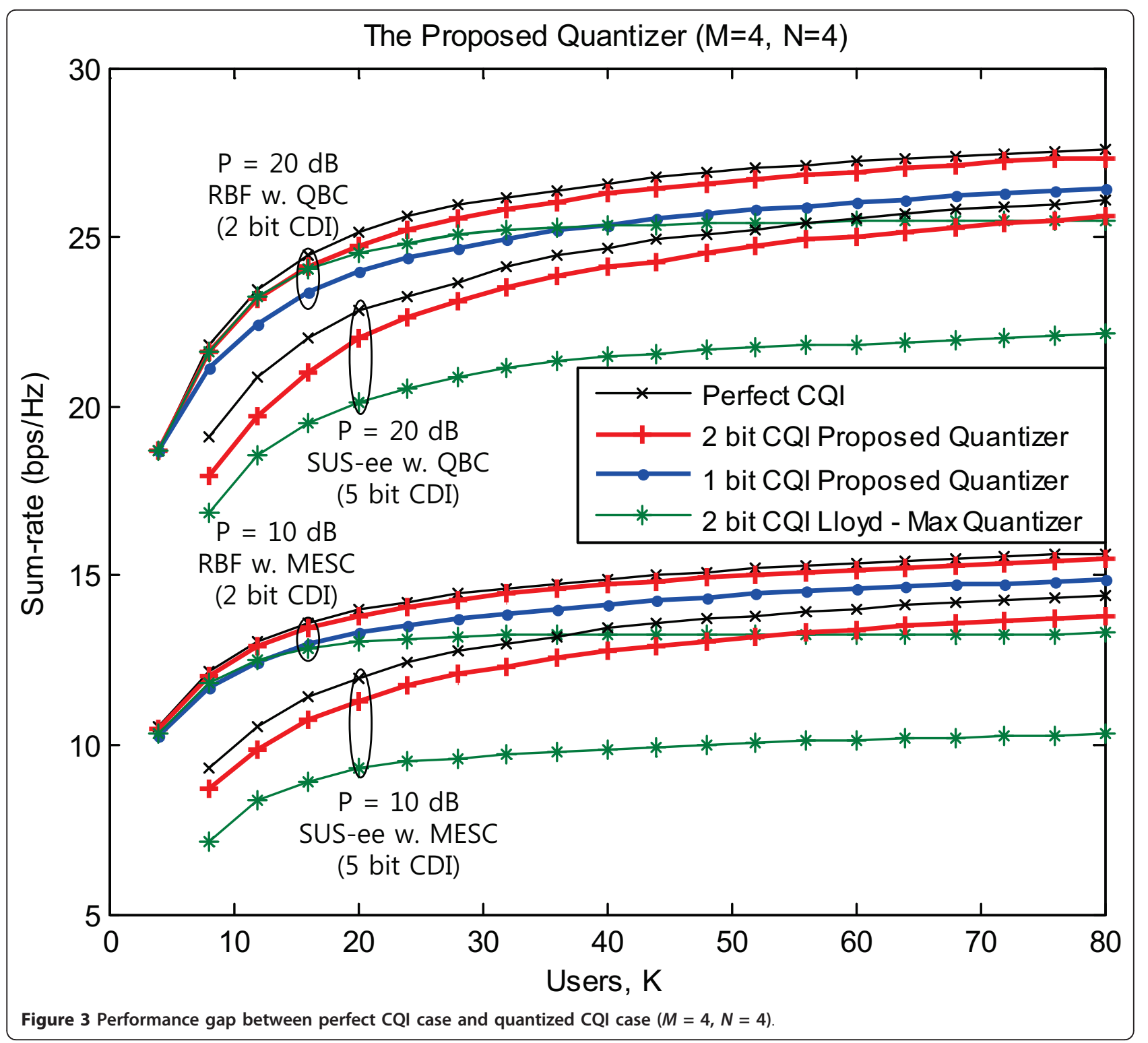

for CQI can be offset by the positive effect from fullorthogonality between the effective channel of each user in the proposed user selection scheme.

On the other hand, when $N$ is much smaller than $M$ $(N=1$ or 2$)$, removing quantization error entirely is not possible. Therefore, RBF system does not guarantee higher throughput than SUS-ee. SUS-ee with QBC or MESC has more codes for antenna combinations than RBF. For this reason, these two systems have additional opportunities to reduce quantization error compared to RBF. In consequence, employing a system which uses large codebook for antenna combinations undoubtedly provides the advantage of increasing the sum-rate of the system.

\section{Conclusion}

In this article, we propose a low-complexity multi-antenna downlink system based on a small-sized CQI quantizer. First, in the proposed system, each user feeds back a CDI and its related CQI collected from $M$ CQIs that are computed according to the every codeword from a codebook of $\log _{2} M$ bit size instead of using a large codebook. In addition, using the extreme value theory, the closed form expression of the expected SINR of selected users is derived. With this formulation, a CQI quantizer is proposed in order to maintain the small-sized feedback system and reflect the sum-rate growth resulting from multiuser diversity. In this work, the sum-rate throughput of the RBF system is obtained by Monte-Carlo simulation 


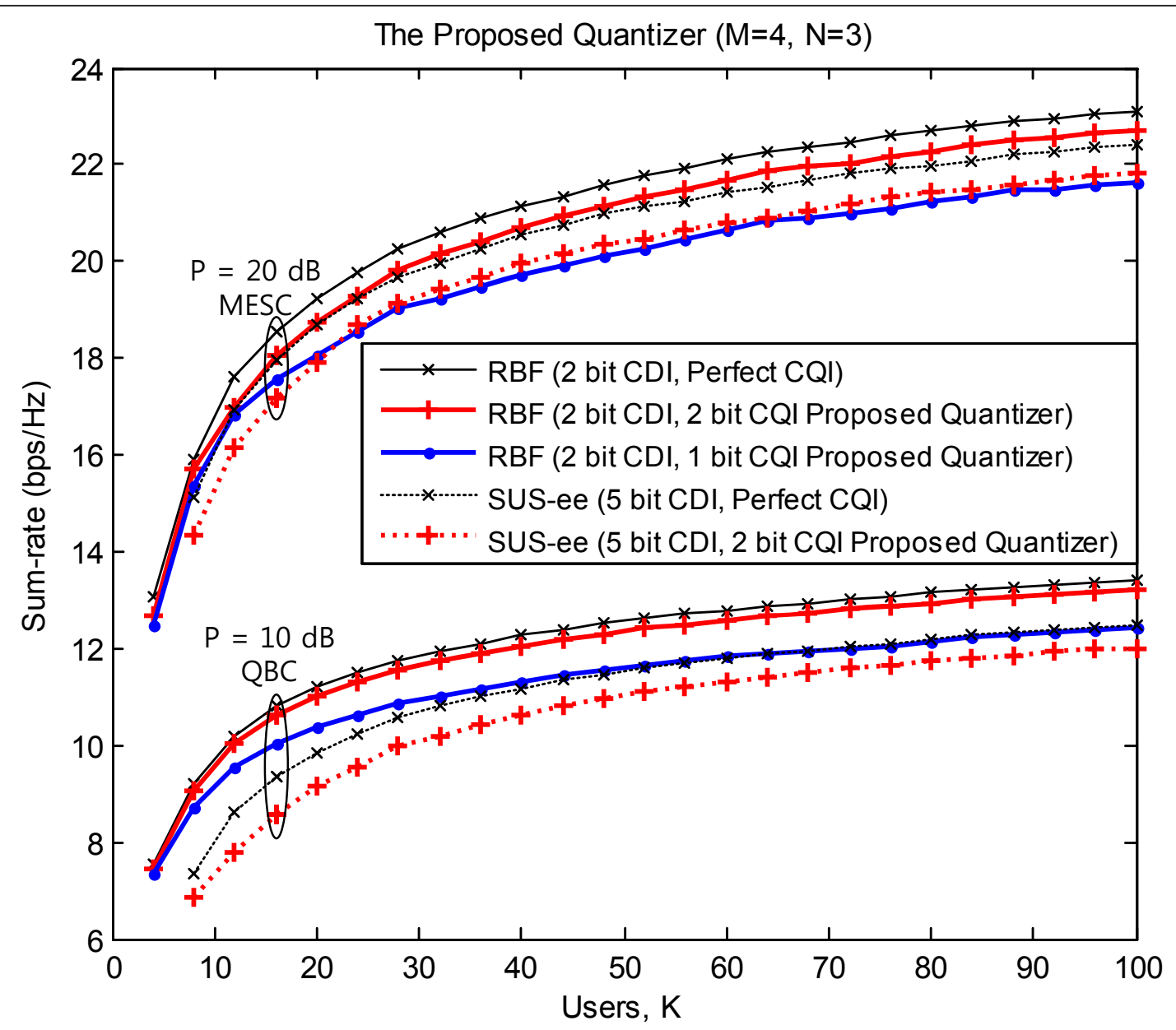

Figure 4 Performance gap between perfect CQI case and quantized CQI case $(M=4, N=3)$.

and is compared to that of a conventional MU-MIMO system based on SUS. Numerical results show that, in the proposed system, the sum-rate can approach the result of SUS-ee with perfect CSIT, outperforming all other systems which are based on SUS-ee under minor amounts of feedback. Furthermore, the results show that performance degradation due to CQI quantization is negligible under the proposed low-bit quantizer. Considering the fairness level of the system, the data rates are distributed quite uniformly among $M$ selected users for RBF, whereas the data rates are weighted too much on the first and second selected users in the SUS-ee algorithm. Finally, the complexity at the BS is reduced as there is no need for precoding multiplication and vector computation in the user selection procedure.

\section{Appendix 1}

\section{Proof of Lemma 1}

Using the distribution of $\left\|\bar{h}_{k, a}^{\text {eff }}\right\| \|^{2}$ and $\sin ^{2} \theta_{k, a}$, the distribution of the interference term is derived. The cdf of $\left\|\bar{h}_{k, a}^{\text {eff }}\right\|^{2} \sin ^{2} \theta_{k, a}$ is described as follows.

$$
\begin{aligned}
& F_{X}(x)=P\left(\left\|\bar{h}_{k, a}^{\text {eff }}\right\|^{2} \sin ^{2} \theta_{k, a} \leq x\right) \\
& =\int_{0}^{\infty} P\left(\sin ^{2} \theta_{k, a} \leq \frac{x}{y}\right) f_{\left\|\bar{h}_{k, a}^{\text {eff }}\right\|^{\mid}}(y) d y \\
& \cong \int_{0}^{\frac{x}{\delta}} f_{\left\|\bar{h}_{k, a}^{\text {eff }}\right\|^{2}}(y) d y+\int_{\frac{x}{\delta}}^{\infty}\left(\begin{array}{c}
M-1 \\
N-1
\end{array}\right)\left(\frac{x}{y}\right)^{(M-N)} f_{\| \bar{h} \overline{k, a}}^{\text {eff }} \|^{2}(y) d y \\
& =1-e^{-\frac{x}{2 \sigma^{2} \delta}}\left[\sum_{k=0}^{m-1} \frac{1}{k !}\left(\frac{x}{2 \sigma^{2} \delta}\right)^{k}\right] \\
& +\left(\begin{array}{c}
M-1 \\
N-1
\end{array}\right) x^{m-1} \frac{1}{\sigma^{2 m} 2^{m} \Gamma(m)} \int_{\frac{x}{\delta}}^{\infty} e^{-\frac{y}{2 \sigma^{2}}} d y \\
& =1-e^{-\frac{x}{2 \sigma^{2} \delta}}\left[\sum_{k=0}^{m-1} \frac{1}{k !}\left(\frac{x}{2 \sigma^{2} \delta}\right)^{k}-\frac{\left(\begin{array}{c}
M-1 \\
N-1
\end{array}\right) x^{m-1}}{\sigma^{2(m-1)} 2^{m-1} \Gamma(m)}\right]
\end{aligned}
$$

(where $m=M-N+1$ ) 


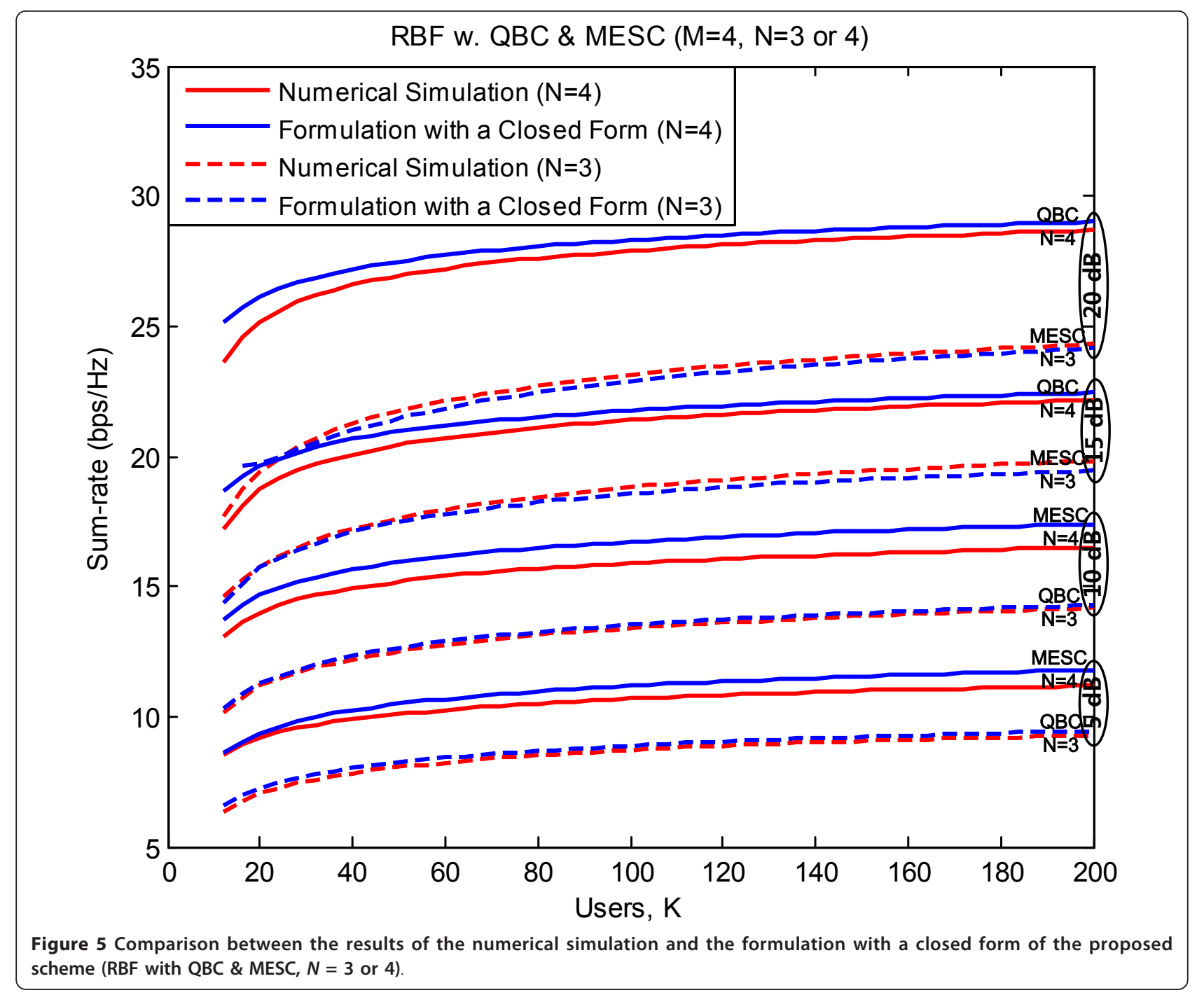

$$
\begin{aligned}
& =\left\{\begin{array}{l}
1-e^{-\frac{x}{2 \sigma^{2} \delta}}\left[1+\frac{x}{2 \sigma^{2}}\left(\frac{1}{\delta}-3\right)\right], \\
1-e^{-\frac{x}{2 \sigma^{2} \delta}}\left[1+\frac{x}{2 \sigma^{2} \delta}+\frac{x^{2}}{2 \cdot 2^{2} \sigma^{4}}\left(\frac{1}{\delta^{2}}-3\right)\right],\left(N=2, \delta=\sqrt{\frac{1}{\left(\begin{array}{c}
M-1 \\
N-1
\end{array}\right)}}\right)
\end{array}\right. \\
& =\left\{\begin{array}{l}
1-e^{-\frac{x}{2 \sigma^{2} \delta}}, \quad\left(N=3, \delta=\frac{1}{\left(\begin{array}{l}
M-1 \\
N-1
\end{array}\right)}\right) \\
1-e^{-\frac{x}{2 \sigma^{2} \delta}}\left[1+\frac{x}{2 \sigma^{2} \delta}\right],
\end{array}\right. \\
& \sim \operatorname{Gamma}\left(M-N, 2 \sigma^{2} \delta\right)
\end{aligned}
$$

\section{Appendix 2}

\section{Proof of Lemma 2}

In Lemma 2, we define both the interference term and the information signal term such as $I_{k}=\left\|\bar{h}_{k, a}^{\text {eff }}\right\|^{2} \sin ^{2} \theta_{k, a}$ and $S_{k}=\left\|\bar{h}_{k, a}^{\text {eff }}\right\|^{2} \cos ^{2} \theta_{k, a}$.
At first, we develop the relation between the joint distribution of $\left(I_{k}, S_{k}\right)$ and that of $\left(\left\|\bar{h}_{k, a}^{\text {eff }}\right\|^{2}, \sin ^{2} \theta_{k, a}\right)$. The relation between the joint distribution of $\left(I_{k}, S_{k}\right)$ and that of $\left(\left\|\bar{h}_{k, a}^{\text {eff }}\right\|^{2}, \sin ^{2} \theta_{k, a}\right)$ are as given by

$$
f_{\|\left.\bar{h}_{k, a}^{\text {eff }}\right|^{2}, \sin ^{2} \theta_{k, a}}(r, w)=|J| f_{I_{k}, S_{k}}(u, v)
$$

where

$$
\begin{aligned}
& u=r w, \quad v=r(1-w) \\
& J=\operatorname{det}\left[\begin{array}{ll}
\frac{\partial u}{\partial r} & \frac{\partial u}{\partial w} \\
\frac{\partial v}{\partial r} & \frac{\partial v}{\partial w}
\end{array}\right]=-r
\end{aligned}
$$

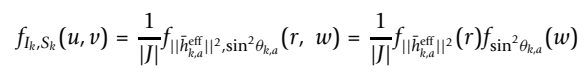

$$
\begin{aligned}
& = \begin{cases}\frac{1}{r} \frac{1}{\sigma^{2 m 2^{m} \Gamma(m)}} r^{m-1} e^{-\frac{r}{2 \sigma^{2}}\left(\begin{array}{c}
M-1 \\
N-1
\end{array}\right)(M-N) w^{m-2},}, 0 \leq w \leq \delta \& r \geq 0 \\
0, & \text { otherwise }\end{cases}
\end{aligned}
$$

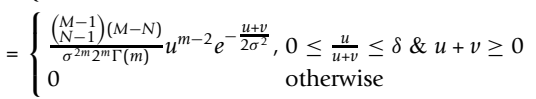




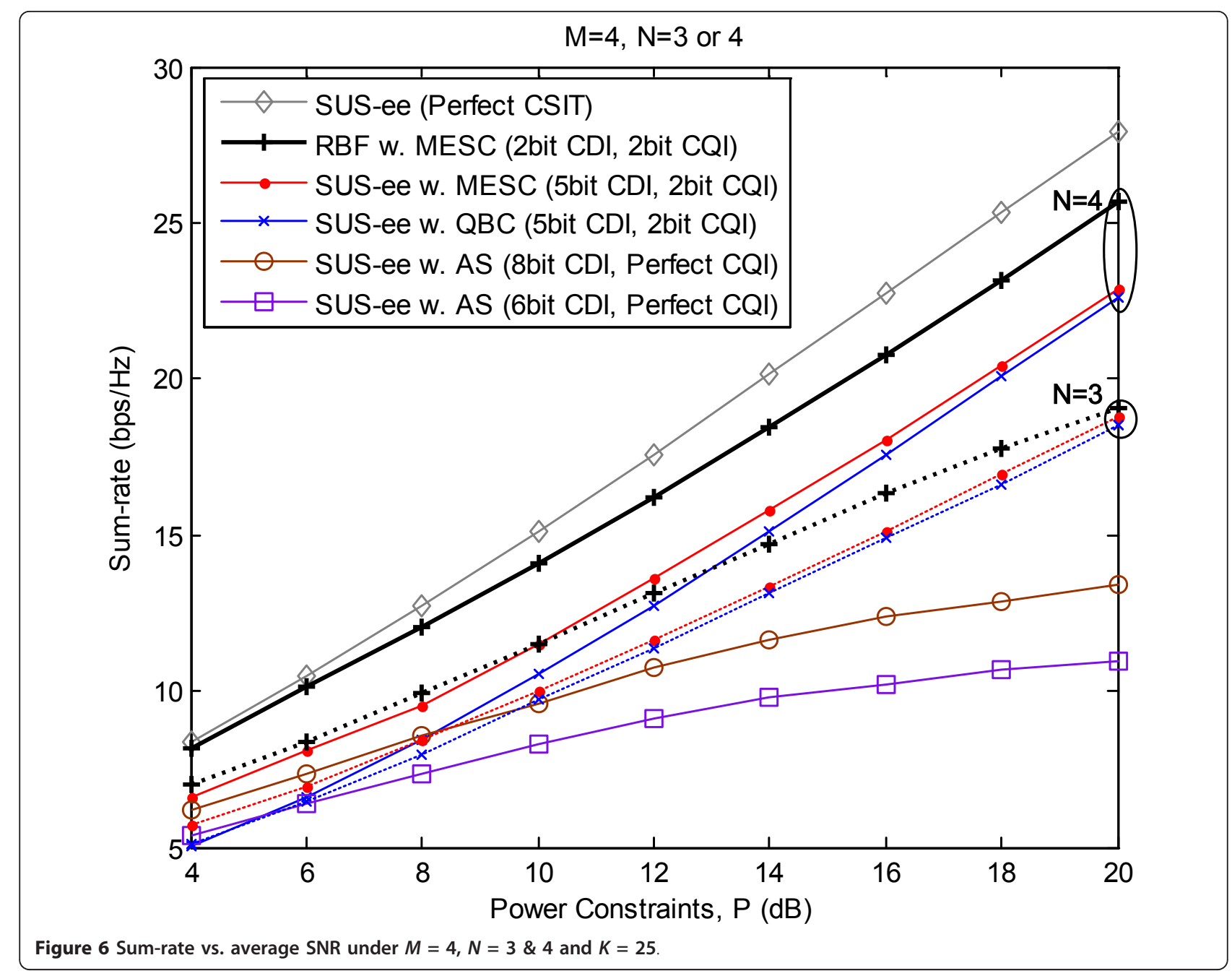

where

$$
\begin{aligned}
& m=M-N+1 \\
& \delta= \begin{cases}\frac{1}{\left(\begin{array}{c}
N-1 \\
N-1
\end{array}\right)}, & (N=M-1) \\
\frac{1}{\sqrt{(N-1)}(N-1)}, & (N=M-2)\end{cases}
\end{aligned}
$$

Then, after defining $I=\delta t Y$ and $S=t(X+(1-\delta) Y)$ where $t=2 \sigma^{2}$, the relation between the joint distributions of $(I, S)$ and that of $(X, Y)$ are derived as follows.

$$
f_{X, Y}(x, y)=|J| f_{I, s}(u, v)
$$

where

$$
\begin{aligned}
& u=\delta t y, \quad v=t(x+(1-\delta) y) \\
& J=\operatorname{det}\left[\begin{array}{l}
\frac{\partial u}{\partial x} \frac{\partial u}{\partial y} \\
\frac{\partial v}{\partial x} \partial \frac{\partial v}{\partial y}
\end{array}\right]=-\delta t^{2}
\end{aligned}
$$

$$
\begin{aligned}
& f_{L, s}(u, v)=\frac{1}{|\nabla|} f_{X, Y}(x, y)=\frac{1}{\delta t^{2}} f_{X}(x) f_{Y}(y) \\
& = \begin{cases}\frac{1}{\delta t} f_{X}\left(\frac{1}{t}\left(u+v-\frac{u}{\delta}\right) f_{Y}\left(\frac{u}{\delta t}\right), \frac{1}{t}\left(u+v-\frac{u}{\delta}\right) \geq 0 \quad \& \quad\right. & \frac{u}{\delta t} \geq 0 \\
0, & \text { otherwise }\end{cases}
\end{aligned}
$$

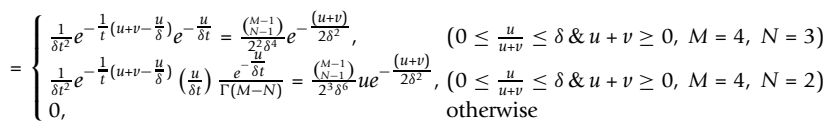

By comparing the equations (31) and (34), we can verify that the joint distribution $f_{I_{k}, S_{k}}(u, v)$ is the same as the joint distribution $f_{I, S}(u, v)$. Therefore, the information signal term $S_{k}$ follows the distribution of $S=t(X+(1-\delta) Y)$ which is described as the sum of two Gamma variables $X$ and $Y$.

\section{Appendix 3}

\section{Proof of Lemma 3}

To derive the cdf of $\gamma_{k, a}$, we define the $\gamma$ using $S$ and $I$ in Lemma $2\left(\gamma=\frac{\rho S}{1+\rho I}=\frac{\rho t X+\rho t(1-\delta) Y}{1+\rho \delta t Y}\right)$. The distribution of $\gamma$ is described as follows. 


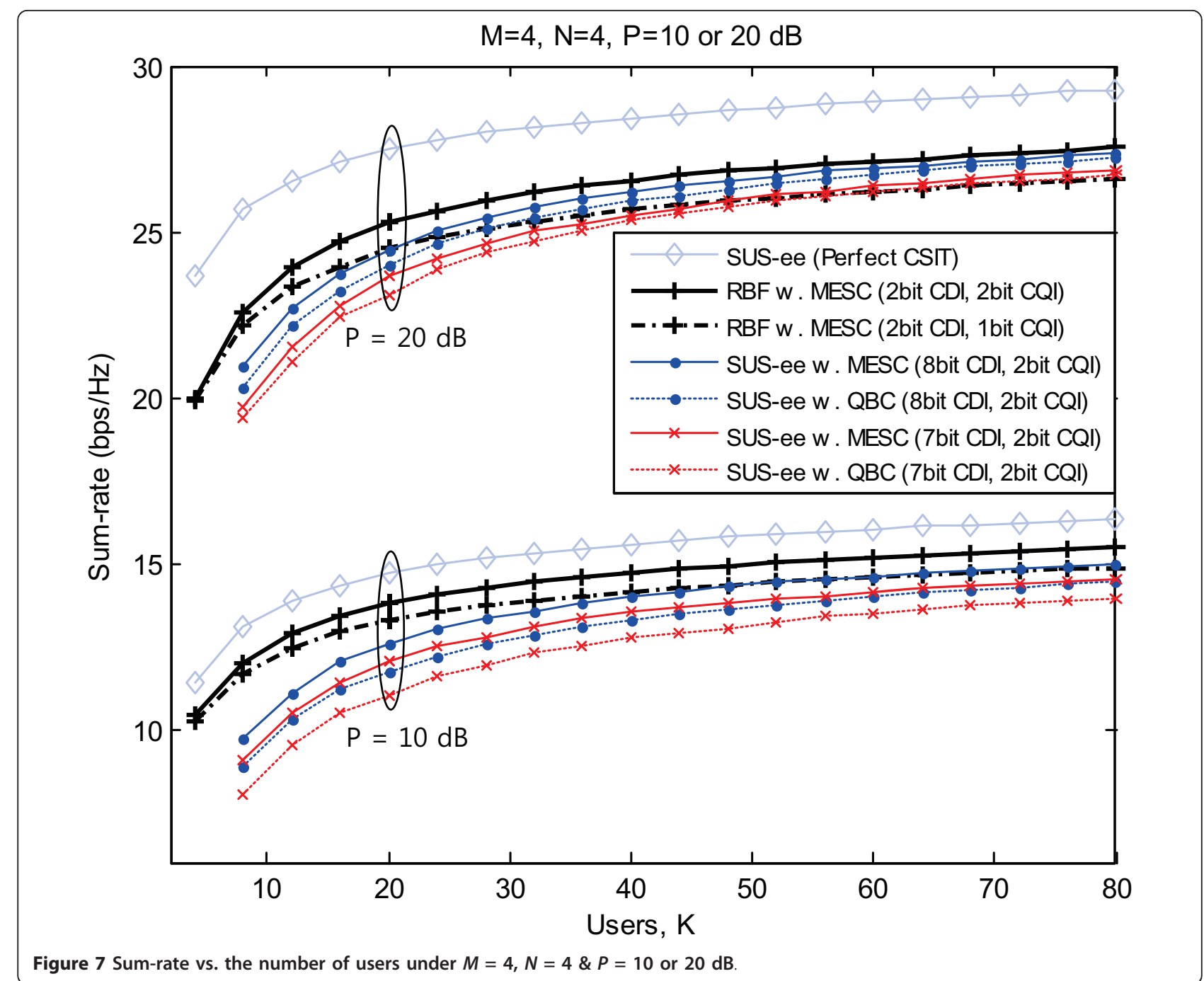

$$
\begin{aligned}
F_{\gamma}(x)= & P(\gamma \leq x)=P\left(\frac{\rho t X+\rho t(1-\delta) Y}{1+\rho \delta t Y} \leq x\right) \\
& =\int_{0}^{\infty} P\left(X \leq \frac{x}{\rho t}+\delta x y+\delta y-y\right) f_{Y}(\gamma) d y \\
= & \int_{0}^{\infty}\left[1-e^{-\left(\frac{x}{\rho t}+\delta x y+\delta y-\gamma\right)}\right] \frac{y^{(M-N-1)} e^{-\gamma}}{\Gamma(M-N)} d y
\end{aligned}
$$

\section{Appendix 4}

Proof of Theorem 1

In this section, we define the relation $\gamma^{\prime}=\frac{\gamma+1}{t \rho} \cdot\left(\gamma=t \rho \gamma^{\prime}-1\right)$ By substituting $\gamma$ with $\frac{\gamma+1}{t \rho}$ the cdf is changed to follow type (iii) distribution in [[18], Theorem 4].

when $\delta x+\delta-1 \geq 0$

$$
\begin{aligned}
F_{\gamma^{\prime}}(z) & =F_{\gamma}(t \rho z-1)=1-\frac{e^{-\frac{1}{t \rho}(t \rho z-1)}}{(t \rho z)^{M-N} \delta^{M-N}} \\
& =1-\frac{e^{\frac{1}{t \rho}}}{(t \rho \delta)^{M-N}} e^{-z} z^{-(M-N)} \doteq 1-\frac{1}{\beta} e^{-z} z^{-\alpha}
\end{aligned}
$$

$=1-\frac{\left(\begin{array}{l}M-1 \\ N-1\end{array}\right) e^{-\frac{x}{2 \sigma^{2} \rho}}}{(x+1)^{M-N}}$ 


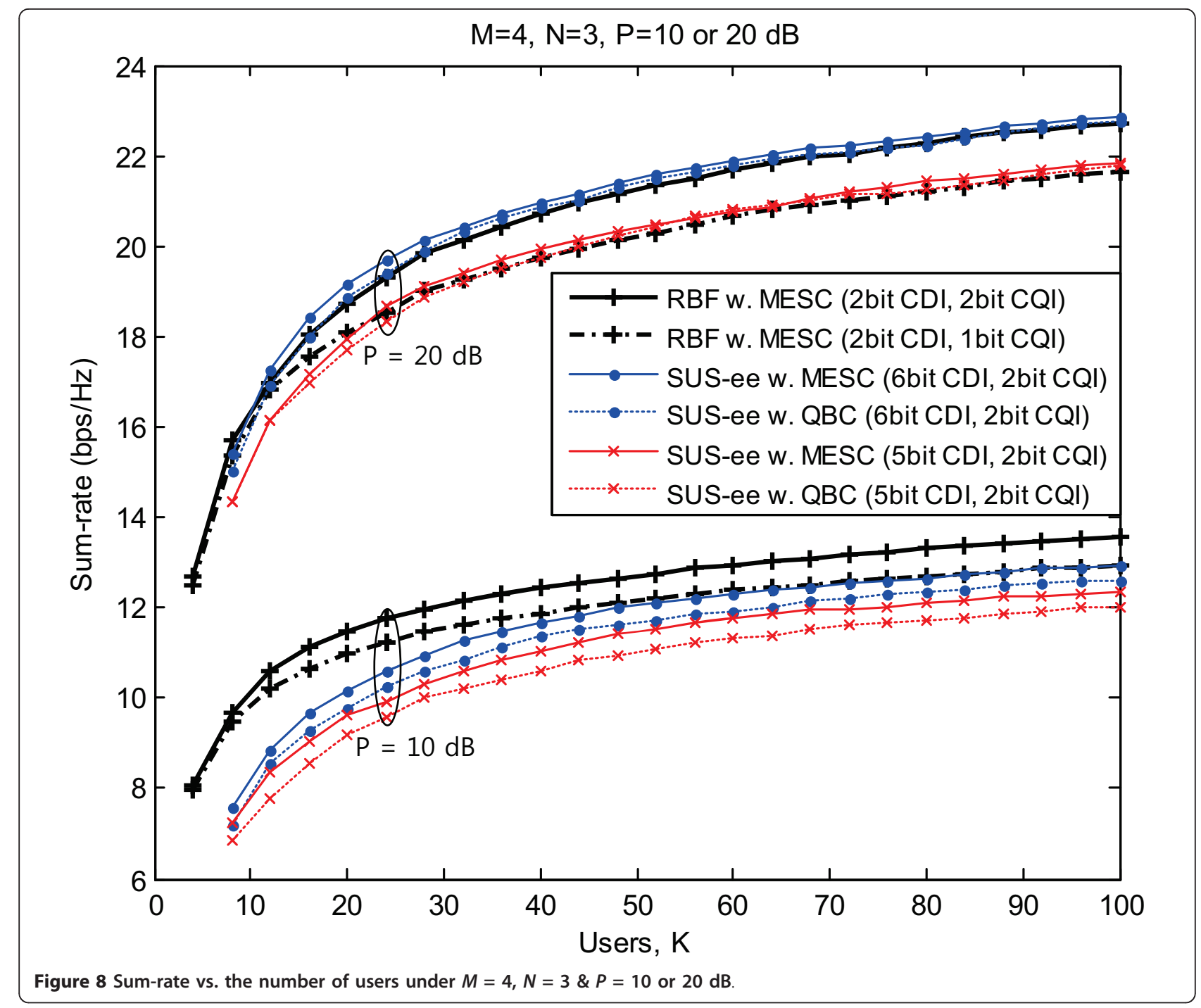

where

$$
\alpha=M-N, \frac{1}{\beta}=\frac{e^{\frac{1}{t \rho}}}{(\delta t \rho)^{M-N}}
$$

Therefore, $\gamma$ can be analyzed using the studies of extreme value theory in order statistics. According to $[18,19]$, the distribution of $\gamma$ satisfies the following inequality

$$
\operatorname{Pr}\left(\left|\gamma_{a: Q_{a}}^{\prime}-b_{Q_{a}}\right| \leq \log \log \sqrt{Q_{a}}\right) \geq 1-O\left(\frac{1}{\log Q_{a}}\right) .
$$

where

$$
\begin{aligned}
& a_{Q_{a}}=1, b_{Q_{a}}=\log \frac{Q_{a}}{\beta}-\alpha \log \log \frac{Q_{a}}{\beta} \\
& =\log \frac{e^{\frac{1}{t \rho}} Q_{a}}{(\delta t \rho)^{M-N}}-(M-N) \log \log \frac{e^{\frac{1}{t \rho}} Q_{a}}{(\delta t \rho)^{M-N}}
\end{aligned}
$$

When $Q_{a}$ is large enough, $\gamma$ satisfies the following approximated formulation,

$$
\begin{aligned}
& \frac{\gamma_{a: Q_{a}}+1}{t \rho}=\gamma_{a: Q_{a}}^{\prime} \cong b_{Q_{a}}=\log \left[\frac{Q_{a}}{(\delta t \rho)^{M-N}}\right] \\
& +\frac{1}{t \rho}-(M-N) \log \left[\log \left[\frac{Q_{a}}{(\delta t \rho)^{M-N}}\right]+\frac{1}{t \rho}\right] . \\
& \gamma_{a: Q_{a}} \cong t \rho \log \left[\frac{Q_{a}}{(\delta t \rho)^{M-N}}\right] \\
& -t \rho(M-N) \log \left[\log \left[\frac{Q_{a}}{(\delta t \rho)^{M-N}}\right]+\frac{1}{t \rho}\right] \\
& \cong 2 \sigma^{2} \rho\left[\log \left[\frac{Q_{a}}{\left(2 \sigma^{2} \delta \rho\right)^{M-N}}\right]\right. \\
& \left.-(M-N) \log \left[\log \left[\frac{Q_{a}}{\left(2 \sigma^{2} \delta \rho\right)^{M-N}}\right]+\frac{1}{2 \sigma^{2} \rho}\right]\right] .
\end{aligned}
$$




\section{Abbreviations}

SINR: signal to interference plus noise ratio; SUS: semi-orthogonal user selection; SUS-ee: semi-orthogonal user selection epsilon expansion; RBF: random beamforming; CQI: channel quality information; CDI: channel direction information; CSI: channel state information; MU-MIMO: multiuser multiple-input multiple-output; DPC: dirty paper coding; BS: base station; MS: mobile station; ZFBF: zero-forcing beamforming; $P U^{2} R C$ : per user unitary and rate control; PMI: preferred matrix index; RVQ: random vector quantization; QBC: quantization-based combining; MESC: maximum expected SINR combiner.

\section{Acknowledgements}

This study was supported by the National Research Foundation of Korea (NRF) grant funded by the Korea government (MEST) (2011-000316) and the Korea Communications Commission (KCC) under the R\&D program supervised by the Korea Communications Agency (KCA) (KCA-2011-0891304003).

\section{Author details}

${ }^{1}$ Department of Electrical Engineering and INMC, Seoul National University, Seoul, Korea ${ }^{2}$ Department of Electronic Engineering, Gachon University, Seongnam, Gyeonggi, Korea ${ }^{3}$ Department of Electronic Engineering, Sogang University, Seoul, Korea

\section{Competing interests}

The authors declare that they have no competing interests.

Received: 25 July 2011 Accepted: 8 February 2012

Published: 8 February 2012

\section{References}

1. P Viswanath, DNC Tse, R Laroia, Opportunistic beamforming using dumb antennas. IEEE Trans Inf Theory. 48(6), 1277-1294 (2002). doi:10.1109/ TIT.2002.1003822

2. M Sharif, B Hassibi, A comparison of time-sharing, DPC, and beamforming for MIMO broadcast channels with many users. IEEE Trans Commun. 55(1), 11-15 (2007)

3. D Gesbert, M Kountouris, RW Heath Jr, CB Chae, T Sälzer, Shifting the MIMO paradigm. IEEE Signal Process Mag. 24(5), 36-46 (2007)

4. M Costa, Writing on dirty paper. IEEE Trans Inf Theory. 29(3), 439-441 (1983). doi:10.1109/TIT.1983.1056659

5. A Goldsmith, SA Jafar, N Jindal, S Vishwanath, Capacity limits of MIMO channels. IEEE J Sel Areas Commun. 21(5), 684-702 (2003). doi:10.1109/ JSAC.2003.810294

6. T Yoo, N Jindal, A Goldsmith, Multi-antenna downlink channels with limited feedback and user selection. IEEE J Sel Areas Commun. 25(7), 1478-1491 (2007)

7. N Jindal, Antenna combining for the MIMO downlink channel. IEEE Trans Wirel Commun. 7(10), 3834-3844 (2008)

8. M Trivellato, F Boccardi, H Huang, On transceiver design and channel quantization for downlink multiuser MIMO systems with limited feedback. IEEE J Sel Areas Commun. 26(8), 1494-1504 (2008)

9. CB Chae, D Mazzarese, N Jindal, RW Heath Jr, Coordinated beamforming with limited feedback in the MIMO broadcast channel. IEEE J Sel Areas Commun. 26(8), 1505-1515 (2008)

10. T Yoo, Sum-capacity, scheduling and multi-user diversity in MIMO broadcast systems. PHD Dissertation, Stanford University (2007)

11. DJ Love, RW Heath Jr, T Strohmer, Grassmannian beamforming for multipleinput multiple-output wireless systems. IEEE Trans Inf Theory. 49(10), 2735-2747 (2003). doi:10.1109/TIT.2003.817466

12. DJ Love, RW Heath Jr, W Santipath, ML Honig, What is the value of limited feedback for MIMO channels?. IEEE Commun Mag. 42(10), 54-59 (2004). doi:10.1109/MCOM.2004.1341261

13. M Sharif, B Hassibi, On the capacity of MIMO broadcast channels with partial side information. IEEE Trans Inf Theory. 51(2), 506-522 (2005) doi:10.1109/TIT.2004.840897

14. Samsung Electronics. Downlink MIMO for EUTRA, 3GPP TSG RAN WG1\#44/ R1-060335

15. W Santipach, ML Honig, Asymptotic performance of MIMO wireless channels with limited feedback, in IEEE Military Communications Conference, Boston, USA, 1, 141-146 (Oct. 2003)
16. CK Au-Yeung, DJ Love, On the performance of random vector quantization limited feedback beamforming in a MISO system. IEEE Trans Wirel Commun. 6(2), 458-462 (2007)

17. JH Winters, J Salz, RD Gitlin, The impact of antenna diversity on the capacity of wireless communication systems. IEEE Trans Commun. 42(234), 1740-1751 (1994)

18. MA Maddah-Ali, MA Sadrabadi, AK Khandani, Broadcast in MIMO systems based on a generalized QR decomposition: Signaling and performance analysis. IEEE Trans Inf Theory. 54(3), 1124-1138 (2008)

19. HA David, Order Statistics (Wiley, New Work, 1980)

20. JG Proakis, Digital Communications, 4th edn. (McGrawHill, New Work, 2001)

21. SP Lloyd, Least squares quantization in PCM. IEEE Trans Inf Theory. 28(2), 129-137 (1982). doi:10.1109/TIT.1982.1056489

doi:10.1186/1687-1499-2012-36

Cite this article as: Song et al:: Low-complexity multiuser MIMO

downlink system based on a small-sized CQI quantizer. EURASIP Journal on Wireless Communications and Networking 2012 2012:36.

\section{Submit your manuscript to a SpringerOpen ${ }^{\circ}$ journal and benefit from:}

- Convenient online submission

- Rigorous peer review

- Immediate publication on acceptance

- Open access: articles freely available online

- High visibility within the field

- Retaining the copyright to your article

Submit your next manuscript at $>$ springeropen.com 